\title{
Development of optical methods for surface and volumetric measurements
}

\author{
Guillaume Gomit ${ }^{1,3,}$, Lionel Thomas ${ }^{1}$, Ludovic Chatellier $^{1}$, Damien Calluaud ${ }^{1}$, Laurent \\ David $^{1}$, Benoit Tremblais ${ }^{2}$, Majdi Khoudeir ${ }^{2}$, Benjamin Bringier ${ }^{2}$, Jean Christophe Dupré ${ }^{1}$, \\ Pierre Morandi ${ }^{1}$, Pascal Doumalin ${ }^{1}$, Fabrice Brémand ${ }^{1}$ \\ ${ }^{1}$ Institut PPRIME, CNRS, Université de Poitiers, ENSMA, France \\ 2 Institut XLIM, CNRS, Université de Poitiers, France \\ ${ }^{3}$ Plateforme CEMOP, Université de Poitiers, CRITT Informatique
}

\begin{abstract}
Optical metrology includes a set of non-contact measurement methods to quantify shapes, surfaces, distances, deformations, displacements or colorimetry. Optical measurement methods have the advantage of being non-intrusive while allowing instantaneous measurements over extended areas. The applications of optical metrology concern all fields of engineering. The paper presents developments related to surface and to 4D measurements (3D displacements + time). Different methods in the fields of solid and fluid mechanics are described and are illustrated by applications. Their applications in different fields for static or dynamic measurements and the limits is discussed in the paper.
\end{abstract}

\section{Introduction}

Optical metrology includes a set of non-contact measurement methods to quantify shapes, surfaces, distances, deformations, displacements or colorimetry. The applications of optical metrology concern all fields of engineering as transport, medical, environment as well as more specific fields such as industrial process monitoring. In this context, the PPRIME and XLIM institutes (University of Poitiers / ENSMA / CNRS) are developing a metrology platform to meet industrial needs. This platform, CEMOP (Centre d'Essais et de Métrologie Optique Picto-charentais), is based on the strong skills in image processing [20] and for optical methods applied to mechanics of both institutes. In the frame of this platform, two research topics are particularly developed: the property measurement of static or dynamic surface and relief, and the 4D measurement (3D displacements + time). The paper presents recent developments related to both measurements.

Surface measurement is a major challenge in industry. Nevertheless, there are many applications: fault detection, surface inspection, face recognition or all applications that require geometric information. Compared to contact method, optical measurement methods have the advantage of being non-intrusive while allowing instantaneous measurements over extended areas. Numerous optical measurement methods have been developed at the Institut PPRIME and at the Institut XLIM for the measurements of solid or fluid surfaces. These methods include stereo-correlation or stereo-refraction for fluid interface [1], solid surface measurement by mark tracking, moiré or structured light projectionas well as multisource photometric measurements [2]. The first part of the paper presents the basic principles of these different methods and recent proposed improvements. Their applications in different fields for static or dynamic measurements and the limits is discussed in the paper.

\footnotetext{
*Corresponding author: guillaume.gomit@univ-poitiers.fr
} 
In a second part, the development of 4D measurement methods, i.e. time-resolved volumetric measurement in the fields of solid and fluid mechanics is presented. For solid mechanic, an optical tomography processing has been developed to investigate non-static mechanical loading in structures with a high spatial resolution. The method is based on a tomographic reconstruction from the scanning of a transparent specimen by a rotating laser sheet coupled with a high-speed camera [3]. The use of this new technique combined with Digital Volume Correlation [4] allows three dimensional displacement measurements and strain field calculation in the bulk of the structure. In fluid mechanics, the development of Tomo-PIV (Particle Image Velocimetry) has shown the possibility of obtaining threedimensional measurements of velocity fields in a volume of significant size. This method is based on the estimation of the displacement of small tracer particles that are advected by the fluid and illuminated by a powerful source of light (laser). Their displacements are recorded by at least 4 digital cameras. Tomographic reconstruction algorithms combined with advanced correlation methods allows the estimation of three-dimensional velocity fields [5].

\section{Surface Measurements}

\subsection{Solid surface}

\subsubsection{Stereo-photogrammetry}

Shape from shading measures the surface shape by analyzing the radiometry of image formation. To improve the results, several acquisitions of the same differently illuminated scene are used and this method is called photometric stereo. The number of acquisitions must be above three and depends on surface complexity as well as geometric and reflectance variations, to obtain a robust and accurate measurement. We note that this number is very dependent on the complexity and the photometric behavior of the surface.

The modeling of the interaction between light and matter is generally divided into two parts. The geometry is expressed by a gradient field whose complexity causes self and cast shadow. And, a BRDF model (Bidirectional Reflectance Distribution Function) that includes the photometric properties of the surface and is generally defined by a diffuse and specular part. For a hemisphere located on a plane and illuminated by a single point source, the figure 1 shows the possible physical phenomena. There are many models of BDRF in the literature that are more or less physically plausible. Unfortunately, only the model proposed by Lambert in 1790 is easy to manipulate in the framework photometric stereo.

The proposed method uses a hierarchical selection of Lambertian reflections to limit the estimation time of the geometric variations. In a first step, for a series of image acquisitions, this selection allows the elimination of shadow which is generated by sources of light onto the surface. This is performed by a local adaptive threshold. This method avoids the determination of an arbitrary threshold. In a second step, the specular reflections are discarded by computing a generalization of the Lambertian error. From this selection, as Woodham has shown, with the Lambertian BRDF model and acquisition of the same scene under angles of different illumination incidence, the partial derivatives of a surface can be measured. This first step produces a gradient field and is obtained by inversion of the BRDF model. Given the gradient fields, a step of integration is needed to estimate the surface height. 


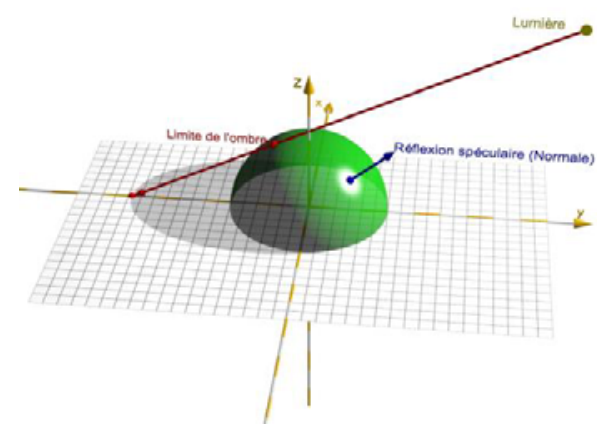

Fig. 1 Interaction between light and a hemisphere surface placed on a plane. The physical properties of the material induce a specular component and the geometry of the surface induces a self and cast shadow.

In order to perform the acquisitions, equipment at low cost can be used. The method allows the estimation of a large area with a great precision in a few seconds. The presented application is performed on a set of surfaces with a great variety of photometric behaviors and geometrical variations. Figure 2 shows the acquisition of five surfaces and the results of the detection of the shadows and of the specular reflections. In this figure, in order to see the details, a square of $10 \mathrm{~mm}$ of side at the center of the acquisitions is presented. We note that the method is not able to correctly identify deep cavities. Indeed, the estimation method uses the interaction of light with the surface to estimate a map. It is evident that areas without light are not considered to be correct. Figure 3 shows the estimation of the height map for all the surfaces of the set.

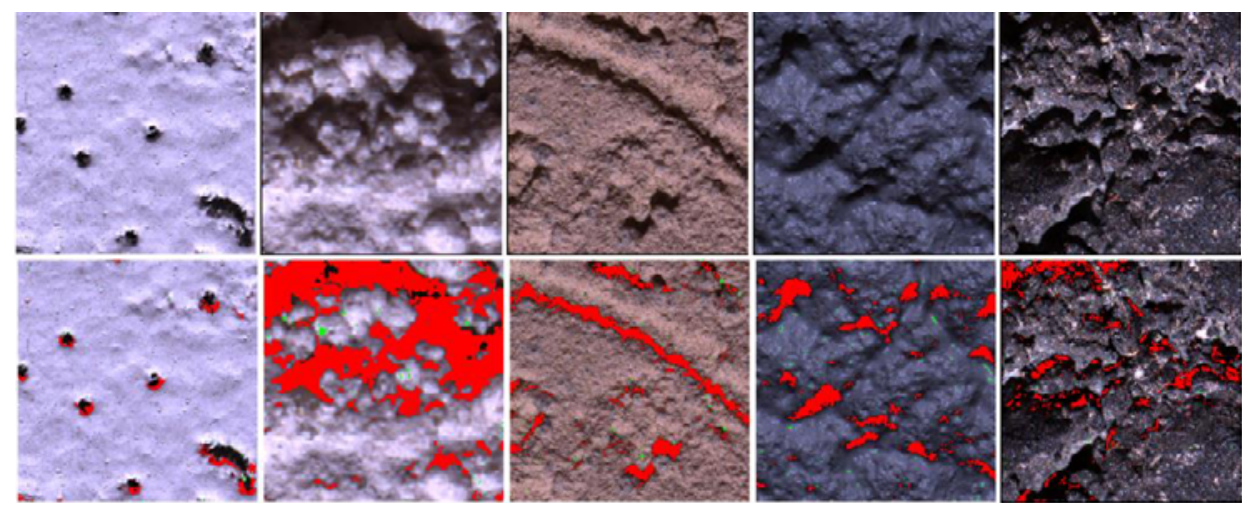

Figure 2 Example of surface acquisitions: detection of shadows (red) and specular reflections (green) 

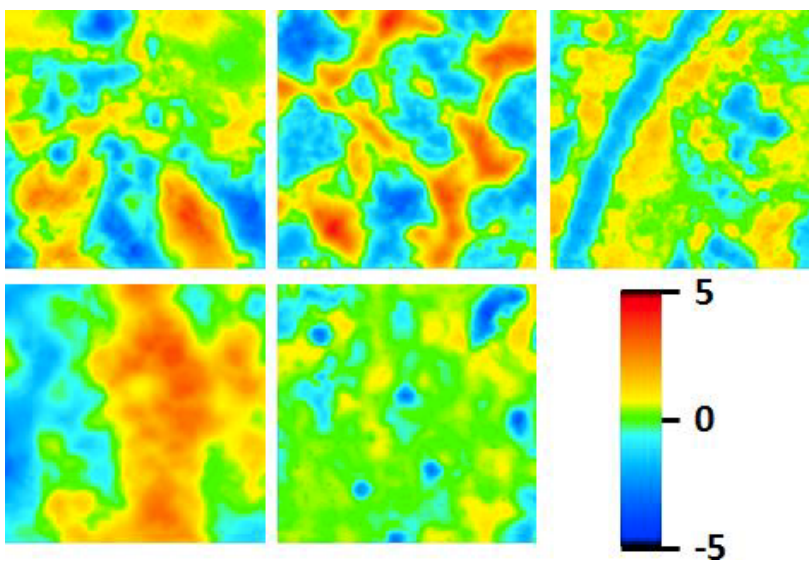

Fig. 3 Estimation of the surfaces (related to Fig. 2). The scale is expressed in mm to represent the heights.

\subsubsection{Projection fringe method}

For relief measurement and out of plane determination of a solid structure submitted to a mechanical loading the projection fringe method can be used. This well-known method [69] consists in projecting a structured light, as a sinusoidal grating of parallel lines, onto the surface of the studied object (figure 4). In order to obtain the relief of the surface, two images are recorded. The first one corresponds to an initial plane which gives the reference and the second one corresponds to the studied specimen. If we assume that the distances camera-object and camera-projector [8-9], are large compared to the measured relief (i.e. with the assumption of parallel beams) the formula given the relief is:

$$
Z=\frac{\Delta \phi}{2 \pi} \cdot \frac{P \text { proj }}{\tan \theta} \text { with } \tan \theta=\frac{d}{h} \text { and } \operatorname{Pproj}=\frac{p}{\cos \theta}
$$

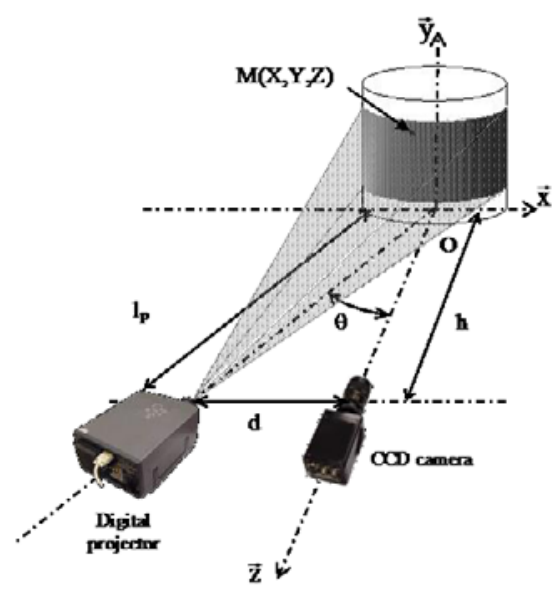

Figure 4

Several tests have been made [6-9], in order to see the influence of this parallel beams criterion. Generally, in solid mechanics, the uncertainty is assessed by a displacement of a 
plan. The accuracy is mainly function to the fringe analysis procedure. The most accurate method consists in recording 8 particular images of the same measurement state [8] and gives a phase precision of $1 / 100$ fringe $(0.04 \mathrm{~mm}$ in considering our optical arrangement $(\mathrm{h}=4 \mathrm{~m}, \mathrm{~d}=1.5 \mathrm{~m})$. Another solution is to use a quasi-heterodyne [8] technique with three images which allows determining the phase with an accuracy of $1 / 40$ fringe $(0.1 \mathrm{~mm})$. These two techniques cannot be used for studying dynamic phenomena where only one image can be recorded at each state. In this case, a single image fringe analysis method like the MPC method [10] must be used. This method determines the fringe pattern morphology with the help of a mathematical model by minimizing a correlation function. The obtained accuracy is about $1 / 20$ fringe $(0.2 \mathrm{~mm}$ is the presented case) .

\subsubsection{Stereo-Mark tracking and stereo-correlation techniques}

These two techniques are based on a stereoscopic vision and the calculation of position of marks for two cameras. These data give by triangulation formulation the $3 \mathrm{D}$ position of the marks. The main differences appear in the images analysis process and type of marks: for mark tracking technique the analysis is done on a series of dots in order to obtain their position and for correlation technique the analysis is currently done on a speckle field (a random distribution of dots). A series of subsets is analysed by a correlation function in order to obtain the relative positions and displacement of this studied zones. The analysis is done thanks to two cameras placed on either side of the normal of the specimen surface $[11,12]$. (figure 5)

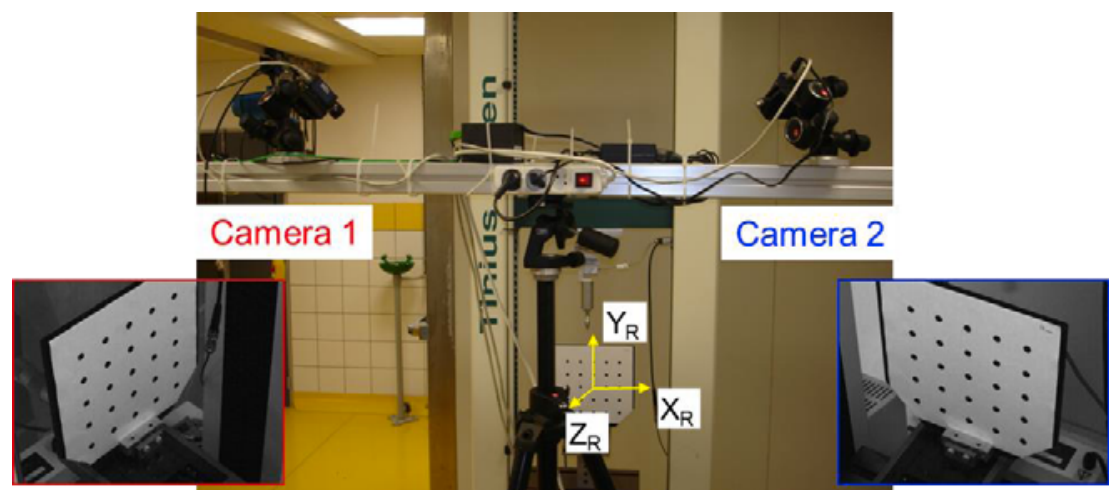

Fig. 5 : set-up for stereo-vision process

In the case of stereo-mark tracking, the coordinates of the markers observed by the two camera $(\mathrm{C} 1$ and $\mathrm{C} 2) \bar{X}_{C 1}$ and $\bar{X}_{C 2}$ are obtained from the geometric center of calculating weighted by the light intensity [13]. A subset is defined around each mark defining the area of calculation. If there is a change in the brightness of the images during the test (due to a change in the lightening of the stage, a change in the surface state ...), the intensity threshold can be modulated for example, depending on the average of values for the calculation area.

The 3D coordinates $\bar{X}_{R}$ of the marks are then given by:

$$
\left\{\begin{array}{l}
\overline{\bar{R}}_{1} \bar{X}_{R}=\overline{\bar{G}}_{1} \bar{X}_{C 1} \\
\overline{\bar{R}}_{2} \bar{X}_{R}=\overline{\bar{G}}_{2} \bar{X}_{C 2}
\end{array}\right.
$$

The position of the cameras are defined by $\overline{\bar{R}}_{1}, \overline{\bar{R}}_{2}$ a rotation matrix (angular positions) and $\overline{\bar{G}}_{1}, \overline{\bar{G}}_{2}$ a transformation matrix (magnificent and position). In order to estimate these matrices, two processes have been developed. The first one consists to solve formula (1) 
with the help of data obtained from images of a grid of marks with known positions of dots. A recording of one image per camera with only one position of the grid is sufficient to obtain theses parameters. Nevertheless, this calibration is useful for small displacement (inferior to 200 times the distance cameras-specimen). For large displacements a second series of images is used with known out of plane displacements to identify a 3D polynomial formulation linking measured displacement and imposed ones.
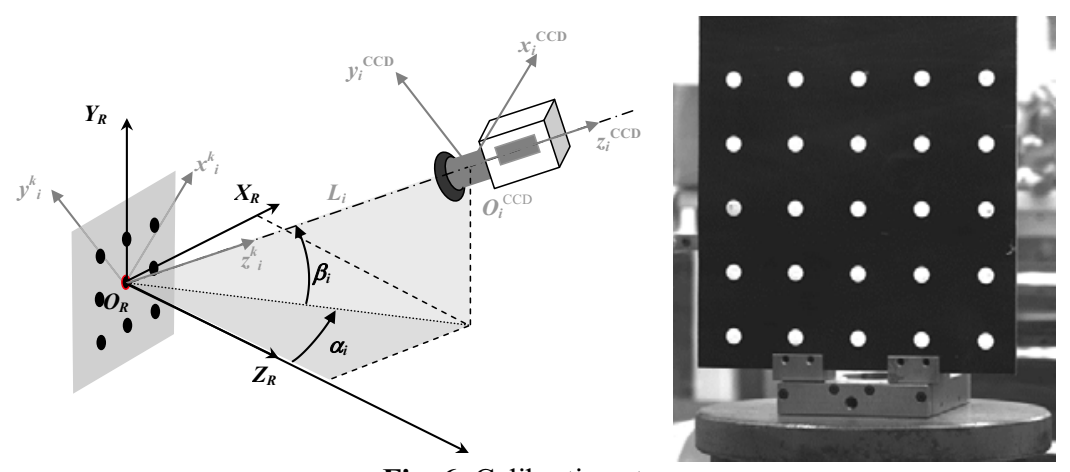

Fig. 6: Calibration step.

Uncertainty is function to the geometric parameter of the device given by the calibration process and function to the image analysis. This late is mainly driven by the dimension of the marks [13]. Experimentally the uncertainty is assessed by a displacement test of a grid. For example for a distance camera-specimen of $2 \mathrm{~m}$, an angle between camera of $26^{\circ}$ and a specimen dimensions of $500 \times 500 \mathrm{~mm}^{2}$. Several out of plane displacements in the range of 0 to $40 \mathrm{~mm}$ with a step of $2 \mathrm{~mm}$ have been done. The bias error between imposed and measured displacement allows us to calculate a standard deviation equal to $0.05 \mathrm{~mm}$ in the 3 directions).

\subsection{Fluid surface}

The measurement of free surface has multiple applications in fluid mechanic. For surface flows, several techniques of surface measurement using optical devices have been developed over the past three decades. These optical measurement methods have the advantage of being non-intrusive while allowing instantaneous measurements over extended areas. The optical measurement methods based on the principle of stereo vision are frequently used in fluid mechanics. This kind of method is based on calibrated multiple views systems and image cross-correlation. In this paper, we present two optical methods that have been used to measure the entire wave field generated by a ship model in a towing tank.

\subsubsection{Stereo-correlation method for fluid surface}

The first method is based on a stereo vision system and on the cross-correlation of surface images. This kind of techniques has often been used in fluid mechanics, for example to measure wave patterns on the surface of the sea. The method of stereo-correlation used here was proposed by [14]. This method requires a tri-dimensional calibration of the cameras. This step of calibration allows to establish the relationship between the 3D world coordinates of a physical point and its corresponding 2D images coordinates.

This method is based on the definition of parametric shape and displacements which are projected on the camera models. These forms are then optimized through a functional that 
correlates the projected image patterns. For the reconstruction surface, the fluid domain is represented by Cartesian coordinates $(x, y, z)$ where $\mathrm{z}=0$ is a reference plane, typically the surface at rest. A series of positions of interest $\left(x_{0}, y_{0}\right)$ in the reference plane is defined. For each of these points of interest, a square area around $\left(x_{0}, y_{0}\right)$ and a parameter, $p_{s}$, defining the height, orientation and curvature of the corresponding surface portion are defined :

$$
p_{s}=\left(h, \frac{\partial h}{\partial x}, \frac{\partial h}{\partial y}, \frac{\partial^{2} h}{\partial x^{2}}, \frac{\partial^{2} h}{\partial y^{2}}, \frac{\partial^{2} h}{\partial y x}\right)
$$

Then, for a point $(x, y)=(x 0+\Delta x, y 0+\Delta y)$ located in a neighbourhood of $(x 0, y 0)$, the height $\mathrm{z}$ can be expressed as the second order bivariate Taylor development of $\mathrm{h}$ in terms of $(\Delta \mathrm{x}, \Delta \mathrm{y})$ :

$$
Z=h+\frac{\partial h}{\partial x} \Delta x+\frac{\partial h}{\partial y} \Delta y+\frac{\partial^{2} h}{\partial x^{2}} \frac{\Delta x^{2}}{2}+\frac{\partial^{2} h}{\partial y^{2}} \frac{\Delta y^{2}}{2}+\frac{\partial^{2} h}{\partial y x} \Delta y \Delta x
$$

For a given parameter $p s$, the image projection of a subset surface around the positions $\left(\mathrm{x}_{0}\right.$, $\mathrm{y}_{0}$ ) can be computed on each camera. Once the images coordinates are evaluated, a bilinear interpolation is used to reconstruct the images of the subset surface. The images corresponding to the surface around $\left(\mathrm{x}_{0}, \mathrm{y}_{0}\right)$ on each camera are then cross-correlated to define the cross-correlation coefficient, $\mathrm{C}(\mathrm{ps})$, to quantify their correspondence. For this technique, which is based on images correlation, polypropylene-floating particles are used to generate a pattern on the surface of the water. The experimental set-up to measure the waves generated by a ship in towing tank in provided in figure 7 . An example of the estimated surface is given in figure 10. For such application, we note that the method is difficult to apply in the wake of the ship where the floating particles are moved sideways.

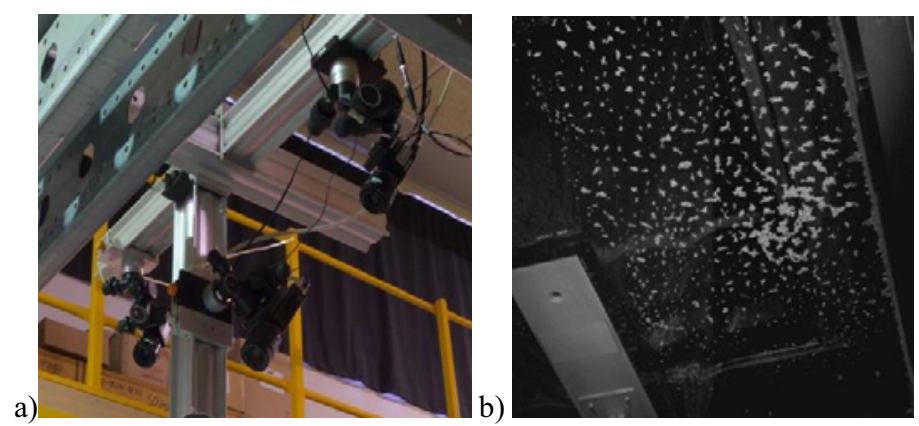

Fig. 7 a) System of stereovision b) Example of images recorded during acquisition

\subsubsection{Stereo-refraction method}

This method is based on the theory of light refraction and on the analysis of the apparent displacement between a reference and refracted images of a laser sheet through the surface [1]. From this apparent displacement, previous methods used the Snell-Decartes law and strong hypothesis on the geometry of the rays issued from the camera to compute the gradient surface. The use of two cameras above the surface to record the apparent displacement of the reference pattern through the interface allows to measure the height and the normal to the surface. The reference pattern is created with a laser sheet that illuminates seeding particles in the flow. Therefore, the technique enables us to measure the topography of the free surface and the instantaneous $2 \mathrm{D}$ velocity in the laser plane.

The principle of the method is provided in figure 8. Given an incident and a refractive vector, the normal vector to the hypothetical surface, $n$, can be computed with equation (2). However, for an incident vector $\mathrm{i}$ and a given point $\mathrm{X}$ on the reference pattern, there are an infinity of couples $P(x, y, z)$ and $\mathrm{n}$ as shown in figure 8 . To close the problem, a second 
point of view is required. This second point of view $\left(C_{2}\right)$, figure 8.c, provides a second incident vector $i_{2}$ reaching point $P(x, y, z)$ supposed on the surface. The apparent displacement view by $C_{2}$, the point $X_{2}$, allows the estimation of the refractive ray $r_{2}$. From incident and refracted rays, the normal vector $n_{2}$ can be computed. The second camera allows to check whether the point $P$ is on the surface or not. If the point $P$ is located on the surface, the two normal vectors $n_{1}$ and $n_{2}$ are equal. A criteria of equality between the normal vectors is used to verify if the hypothetical point $P$ actually belongs to the surface.

a)

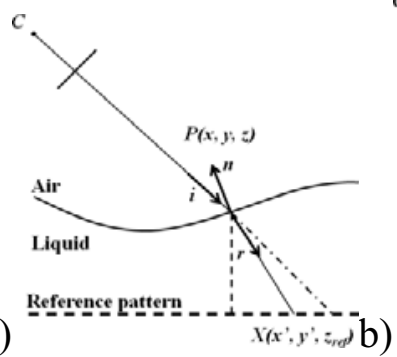

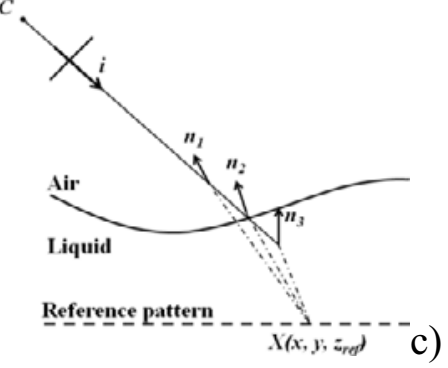

Fig. 8 Light refraction

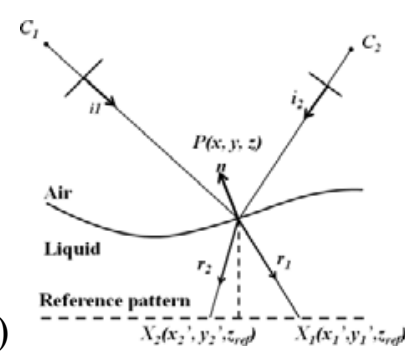

) The principle of the method proposed here is based the apparent displacement of a random
pattern located below the surface viewed by two cameras above the surface. In experimental set-up (figure 9), the random pattern is generated with a laser sheet, parallel at the surface at rest, that illuminates seeding particles in the flow. Therefore, this pattern is similar to the pattern recorded during PIV measurements. A camera located under the tank provides the reference pattern, i.e. the non-deformed pattern. The two cameras above the surface provide the deformed state of the laser sheet through the interface. An adapted PIV algorithm for the refracted images is then used to determine the apparent displacement between the cameras located above the surface and the reference camera. The reconstruction of the surface requires the calibration of the three cameras in the same physical volume of reference. Moreover, as note previously, this technique enables us to measure the topography of the free surface and the instantaneous $2 \mathrm{D}$ velocity in the laser plane as show in the example of figure 11.b.

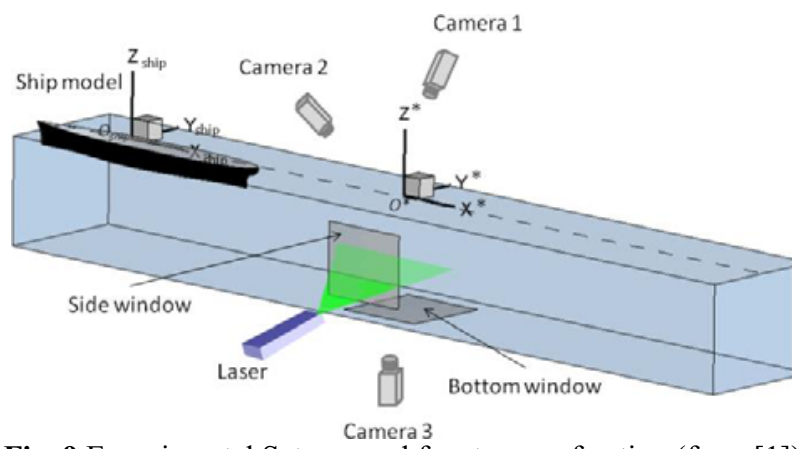

Fig. 9 Experimental Set-up used for stereo-refraction (from [1]) 
a)

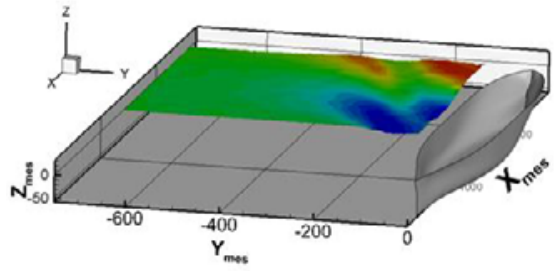

b)

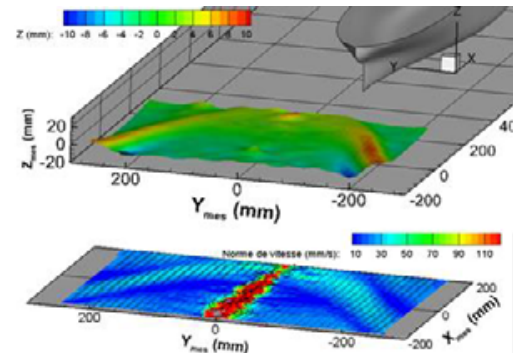

Fig. 10 Instantaneous measurements: a) Free surface measured with stereovision system b) Free surface and velocity field measured by "stereo-refraction"

\section{4D Measurements}

The study of complex mechanical phenomenon, whether in the fields of solid and fluid mechanics, can require more than surface or $2 \mathrm{D}$ measurements. Therefore, time-resolved volumetric measurement methods have been developed.

\subsection{Solid application}

This section concerns an optical scanning tomography technique developed for timeresolved measurement of kinematic fields in the whole volume of transparent model structures. Previously, 3D photoelasticity for stress measurement and Optical Scanning Tomography (OST) $[4,15]$ for strain measurement were developed. Nevertheless this late method is not adapted for non-static studies; because the principle of this technique consists in scanning by a plane laser beam a transparent specimen including thin particles. At each position of the beam, a two-dimensional image of the illuminated section is recorded. The movement is performed thanks to a translation stage monitored by a computer $[4,15]$ or oscillating mirror. Due to inertia phenomenon, with these devices, we cannot record a large specimen (several centimetres) in a recording time lower than one second. Consequently, we have developed a new technique with a scanning by rotating the plane laser beam, named Optical Rotation-Scanning Tomography (ORST).

A laser beam (figure 11) passes through a cylindrical lens producing a plane laser beam. This lens rotates along its optical axis. The specimen is placed above this axis given a small rotation angle range and a minimum of overlapping of illuminated particles along the view axis. High speed camera is placed perpendicularly to the optical axis of the lens. As the recorded images correspond to a non-parallel series of slice, a specific reconstruction process is necessary to calculate grey levels of voxels and obtain the volume. Parameters of reconstruction are determined by a two phases calibration procedure: (i) analysis of images obtain from a translation of a known grid of marks and (ii) analysis of images of the rotated laser beam with imposed known translation of a plan.

The rotary optical device is constituted by a cylindrical lens activated by a direct current motor. An angular encoder with a resolution of $100 \mu \mathrm{rd}$ is fixed around the lens to synchronize the lens angulation and camera acquisition. For the following test the rotation speed was about $6 \mathrm{tr} / \mathrm{min}$ and camera frame rate was $1200 \mathrm{frame} / \mathrm{s}$. So a volume is obtained in $0.2 \mathrm{~s}$, optical magnification was about $60 \mu \mathrm{m} / \mathrm{voxel}$,

To assess uncertainty, a test of subvoxel rigid body translation is realized. The specimen is scanned for each translation of $1 / 10$ voxel, the three directions $(x, y, z)$ of the space are studied. The translation is achieved with a manual micrometer translation stage. After having reconstructed 3D images, displacements are calculated by Digital Volume 
Correlation method [15]. The subset size is set to 31 voxels 3 and calculi are performed on a grid containing $7 \times 7 \times 7=343$ correlation points. The main conclusion is that the uncertainty is closed to the one obtain in 2D Correlation (DIC) [16]. Moreover, the mean error versus imposed displacement shows the well-known s-curve given by DIC grey level interpolation.

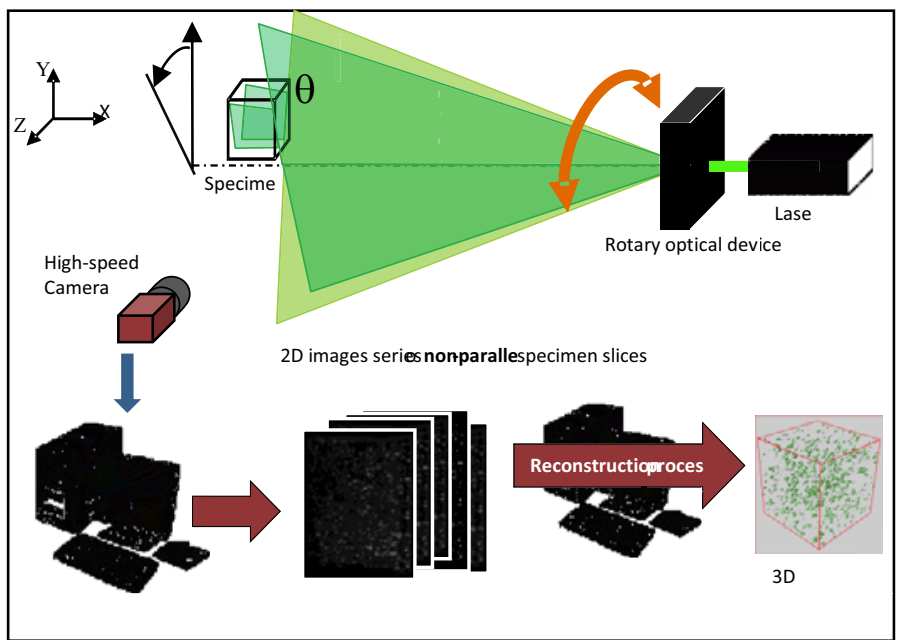

Fig. 11 Device and methodology of ORST

\subsection{Fluid application}

The principle of the Tomo-PIV is based on the computation of the velocity vector field of a flow from the displacement of the particles recorded on several images. Particles are seeded in the flow and are illuminated by a thick laser sheet which defines the volume of interest. The light scattered by the particles is captured by several high-resolution digital cameras from different viewing angles (usually 4 - see figure 12). The information on the line of sight of each pixel in a camera, through the examined volume, is described by a polynomial approximation made from a $3 \mathrm{D}$ calibration procedure. Thus, the projection images set captured at a given time are supplied to a $3 \mathrm{D}$ tomographic reconstruction algorithm to reconstruct a volume of $3 \mathrm{D}$ particles. Therefore, tomographic reconstruction algorithms combined with advanced correlation methods allows the estimation of threedimensional velocity fields [5].

Here, the instantaneous fields of velocity and material acceleration are provided by the recently introduced multi-frame PIV method, fluid trajectory evaluation based on ensemble-averaged cross-correlation [17] (FTEE). FTEE is capable of evaluating the most appropriate Lagrangian fluid trajectory from multiple particle images. The trajectory which maximizes an ensemble averaged cross-correlation along the trajectory is regarded as the solution. The fluid motion at each grid point is modeled as the polynomial function. In FTEE, the cross-correlation maps are regarded as probability maps of the trajectory at the discrete time steps, and the resulting trajectory signifies the most probable fluid motion. It has been reported that the random error of FTEE is less than other TR-PIV methods. Given the velocity and acceleration, recent method allows the estimation of pressure in the flow. The principle of the pressure estimation method is to combine the measured velocity/acceleration data with the equations governing the flow. An example of the measured flow and pressure around a flapping wing [19] is shown in figure 13. 


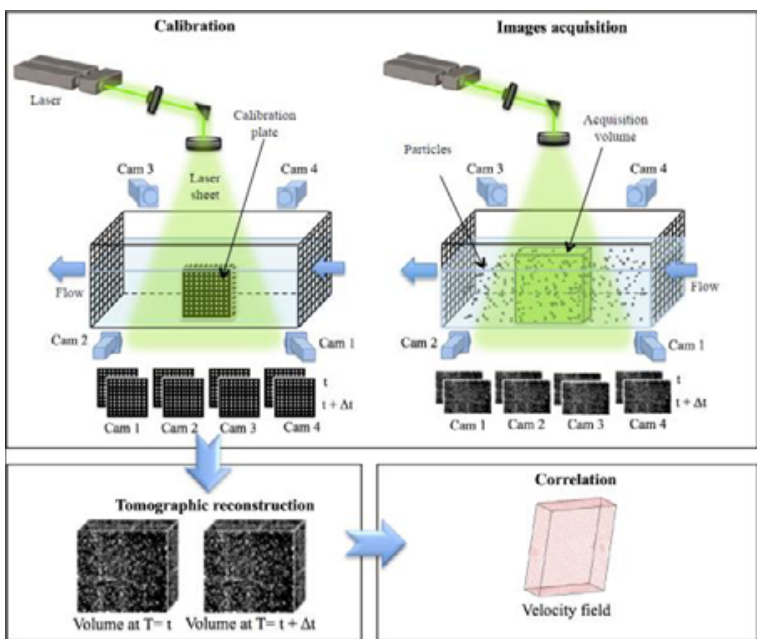

Fig. 12 Principle of tomographic-PIV [18]

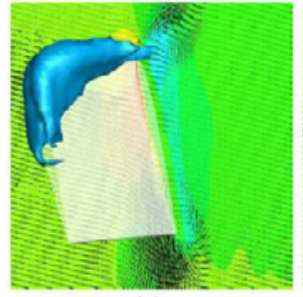

(a)

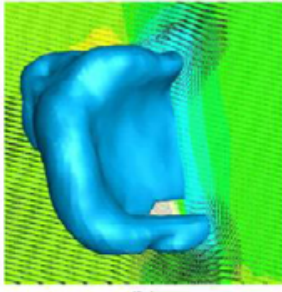

(b)

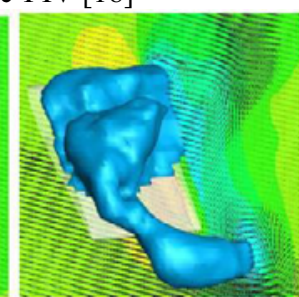

(c)

Cp: $\begin{array}{rrrrrrrrrrrrrrrrr}-4 & -3.5 & -3 & -2.5 & -2 & -1.5 & -1 & -0.5 & 0 & 0.5 & 1 & 1.5 & 2 & 2.5 & 3 & 3.5 & 4\end{array}$

Fig. 13 Flow around a flapping wing from [19]

\section{Conclusion}

Numerous optical measurement methods for the measurements of solid or fluid surfaces have been presented. These non-contact methods allow accurate measurements on large areas for solid and fluid interfaces. Moreover, time-resolved volumetric measurement methods have been developed to analysis complex 3D mechanics problem in the fields of solid and fluid mechanic. In perspective to use such optical methods in industrial cases, detailed studies on uncertainty are needed.

\section{Acknowledgements}

The current work has been funded partially by the $14^{\text {th }}$ Contrat Plan Etat Region and the associated FEDER project No P-2016-BAFE-227.

\section{References}

1. G. Gomit, L. Chatellier, D. Calluaud, L. David. Exp. in Fluids, 54-1540, (2013)

2. A. Bony, B. Bringier and M. Khoudeir, J. Opt. Soc. Am. A, Vol. 30, No. 3, (2013).

3. P. Morandi , F. Brémand ,P. Doumalin ,A. Germaneau ,J.C. Dupré, , Optics and Lasers in Eng., Vol. 58, Pages 85-92, (2014) 
4. A. Germaneau; P. Doumalin; J.C. Dupre, NDT and E International, 41 (6), (2008)

5. L. Thomas, B. Tremblais, L. David, Meas., Sci. and Tech. 25:3, 035303 (2014)

6. L. Pirodda, Opt. Eng. 21:640-9, (1982)

7. P.Théocaris, Pergamon press Ltd, Headington hill hall, Oxford, (1969).

8. C. Brèque, J.C Dupre., F. Brémand, Optics and Lasers eng., (2003).

9. F. Brémand, Opt Laser Eng;21:pp. 49-60. (1994)

10. E. Robin, V. Valle, Applied Optics, vol 43, n²2, pp 4355-4361 (2004)

11. F. Brémand, M. Cottron, P. Doumalin, J.C. Dupré, A. Germaneau, V. Valle, Techniques de l'ingeniéur, 2. R1850 (2011)

12. A. Germaneau, P. Doumalin, J.C. Dupré, , C. Brèque, F. Brémand, S. D’Houtaud and P. Rigoard, Eur. Phys. J, (2010).

13. N. Bretagne, V. Valle and J.-C. Dupré, NDT \& E International, Vol 38/4,(2005)

14. Chatellier, L., Jarny, S., Gibouin, F., \& David, L. Exp. in fluids, 3(54), 1-15. (2013)

15. A. Germaneau, P. Doumalin, J.C. Dupre, 43, 207-218 (2007)

16. J. C. Dupré, P. Doumalin, H. A. Husseini, A. Germaneau and F. Brémand, Strain, Volume 51, Issue 5, (2015)

17. Y. J Jeon, L. Chatellier, L. David, Exp. in fluids, 55(7), 1766 (2014)

18. R. Ben Salah, PhD thesis, University of Poitiers, (2015)

19. T. Tronchin, L. David, A. Farcy Exp. in fluids, 56:7 (2015)

20. Tremblais B., David L., Arrivault D., Dombre J., Chatellier L., and Thomas L., "Slip :

Simple library for image processing (version 1.0)," http://www.sic.sp2mi.univ-

poitiers.fr/slip/, 2010. 